\title{
Folle machine ou solide relation « living apart together »?
}

Le rôle des métaphores dans la perception citoyenne du fédéralisme belge

A crazy machine or a strong "living apart together" relationship? The role of metaphors in citizens' perception of Belgian federalism ¿La máquina loca o la solidez de la relación « living apart together "? La función de las metáforas en la percepción ciudadana del federalismo belga

\section{Julien Perrez et Min Reuchamps}

\section{OpenEdition}

Journals

Édition électronique

URL : https://journals.openedition.org/mots/22156

DOI : $10.4000 /$ mots. 22156

ISSN : $1960-6001$

Traduction(s) :

A crazy machine or a strong "living apart together" relationship? - URL : https:// journals.openedition.org/mots/22171 [en]

Éditeur

ENS Éditions

Édition imprimée

Date de publication : 30 novembre 2015

Pagination : 125-145

ISBN : 978-2-84788-776-1

ISSN : 0243-6450

Référence électronique

Julien Perrez et Min Reuchamps, "Folle machine ou solide relation « living apart together »? », Mots. Les langages du politique [En ligne], 109 | 2015, mis en ligne le 30 novembre 2017, consulté le 23 avril 2022. URL : http://journals.openedition.org/mots/22156 ; DOI : https://doi.org/10.4000/mots.22156 


\section{Folle machine ou solide relation " living apart together " ? Le rôle des métaphores dans la perception citoyenne du fédéralisme belge}

À la suite de l'ouvrage fondateur de Lakoff et Johnson (1980), bon nombre de travaux ont été consacrés à l'étude des métaphores, non pas en tant que figures rhétoriques, mais comme des outils conceptuels structurant une réalité complexe. Pour les partisans de la Conceptual Metaphor Theory (CMT), les métaphores jouent un rôle majeur dans les processus de perception et de catégorisation d'entités abstraites et, ce faisant, elles nous permettent de structurer notre compréhension des processus complexes ${ }^{1}$.

Bien que les métaphores conceptuelles soient présentes dans tous les domaines de la vie quotidienne, le champ politique est un domaine où les métaphores jouent un rôle prépondérant. Comme le suggère Semino (2008, p. 90, notre traduction) : «L'emploi de métaphores est particulièrement nécessaire en politique, dans la mesure où le domaine politique est un domaine abstrait et complexe, et dans la mesure où les métaphores peuvent contribuer à simplifier ces complexités et rendre accessibles ces abstractions. » L'importance des métaphores dans le domaine politique a aussi été démontrée par Charteris-Black (2011, p. 28, notre traduction), qui souligne leur rôle dans la construction des représentations mentales des questions politiques et qui suggère que leur pouvoir réside dans leur capacité à «activer des associations émotionnelles qui contribuent à la création de mythes». Le besoin d'approfondir les recherches sur l'impact politique des métaphores a par conséquent souvent été souligné : «Si les métaphores se trouvent au cœur du processus

1. Les auteurs tiennent à remercier Tina Krenmayr, les évaluateurs anonymes et les membres du comité éditorial, et en particulier Chloé Gaboriaux, de la revue Mots. Les langages du politique, pour leurs remarques constructives sur la version préliminaire de cet article. La responsabilité de toute erreur qui subsisterait dans ce texte incombe évidemment aux auteurs. Cette recherche a été réalisée avec le soutien financier des Fonds spéciaux pour la recherchede l'Université catholique de Louvain (UCL) et de l'Université de Liège (ULg), Belgique, et par le Fonds de la Recherche Scientifique - FNRS sous la référence PDR-T.1036.15.

Université de Liège

Julien.Perrez@ulg.ac.be

Université catholique de Louvain

Min.Reuchamps@uclouvain.be

Mots. Les langages du politique $\mathrm{n}^{\circ} 109$ novembre $2015 \bullet 125$ 
de cadrage cognitif, elles sont cruciales pour l'étude du domaine politique» (De Landtsheer, 2009, p.60, notre traduction).

Dans cette perspective, plusieurs chercheurs en linguistique et en science politique se sont lancés dans l'étude des métaphores dans le domaine politique (voir Bougher, 2012). Cependant, jusqu'à présent, l'accent a été principalement mis sur les discours des élites politiques (voir, par exemple, Charteris-Black, 2011). Bien que l'analyse des métaphores utilisées par les élites permette de comprendre comment les métaphores façonnent le débat politique (L'Hôte, 2010, 2012 ; Mercenier et al., 2015), les discours citoyens sur la politique devraient également être étudiés. En effet, hormis quelques exceptions, comme par exemple une recherche sur les perceptions du terrorisme par le grand public, d'une part, et par les experts, d'autre part (Cameron, Maslen, 2010), une telle approche a été largement négligée, ainsi que l'observe Bougher (2012, p.149, notre traduction) : «Alors que les recherches sur les métaphores dans le discours politique ont foisonné au cours des dernières années, cette focale sur la communication des élites a laissé inexploré le rôle plus large des métaphores comme un outil de raisonnement pour les citoyens. »

Plus spécifiquement, la recherche sur les discours des citoyens peut mener à deux types d'enseignements : d'un côté, elle permet d'évaluer dans quelle mesure les métaphores produites par les élites politiques sont intégrées dans le raisonnement politique du citoyen et, d'un autre côté, elle offre également l'opportunité de saisir comment les citoyens «produisent leurs propres métaphores pour comprendre leur environnement politique»(Bougher, ibid.).

Ainsi, cette contribution a précisément pour objectif d'adopter une approche «bottom-up», basée sur des groupes de discussion, analysant comment les citoyens perçoivent le système politique belge, et plus précisément d'évaluer le rôle que jouent les métaphores dans cette perception. Pays fortement divisé s’il en est, la Belgique offre un cas d'étude intéressant pour aborder cette question, puisque la transformation de son État unitaire en un État fédéral s'est réalisée dans un climat de tensions entre et au sein des deux principaux groupes linguistiques/communautés : les néerlandophones/Flamands et les francophones/Wallons (Perrez, Reuchamps, 2012). En raison de la prégnance de cette question, particulièrement de 2007 à 2011, lorsque la Belgique était plongée dans une longue crise politique (Deschouwer, 2012 ; Deschouwer, Reuchamps, 2013), se pose la question de savoir si les métaphores sont présentes dans le discours des citoyens à propos du fonctionnement et de l'avenir de leur pays. 


\section{Données et méthodes}

\section{Corpus citoyens}

Alors qu'il est fréquent d'étudier les discours des élites politiques, il est moins commun de se concentrer sur les discours des citoyens. En tant que tels, les discours publics des citoyens n'existent que rarement. Néanmoins, les citoyens parlent souvent de politique. Pour saisir ces discussions, deux méthodes différentes sont possibles: l'une est d'enregistrer des conversations authentiques de la vie quotidienne, par exemple des discussions familiales ou des forums en ligne; une autre méthode consiste à organiser des groupes de discussion. La pertinence de ces outils a notamment été mise en évidence par De Cillia et al. (1999, p.152-153) :

The method of the "focus-group discussion" [...] offers a very promising tool for ethnographic research in Critical Discourse Analysis. It enables one partially to study the recontextualization and transformation of specific political concepts and identity narratives which are expressed by politicians, taught in educational systems (e.g. by teachers and in schoolbooks), promoted in the mass-media, etc., and which are expressed in everyday situations and interactions.

Bien que cette méthode offre des perspectives prometteuses pour l'étude des métaphores, elle n'a que très rarement été mise en œuvre (voir, pour quelques exceptions, par exemple Cameron, 2007; Cameron, Maslen, 2010).

Pour étudier les métaphores dans les discours citoyens, notre recherche repose sur les données récoltées lors de huit groupes de discussion composés de six à neuf personnes et organisés après les élections fédérales de 2007, en pleine crise politique. Quatre se sont tenus à Liège (Belgique francophone) et quatre à Anvers (Belgique néerlandophone). Pendant quatre heures, les participants, venus d'horizons divers et aux préférences politiques variées, ont discuté du fédéralisme belge, avec d'autres citoyens ainsi qu'avec des politiciens et des experts (Reuchamps, 2011; Reuchamps, Perrez, 2012). Les échanges avec deux politiciens (un francophone et un néerlandophone, de deux partis différents) et deux experts (un professeur d'université et un journaliste) avaient pour but d'offrir aux citoyens différents éclairages sur la problématique. Les participants se sont d'abord réunis en petits groupes pour une première séance de discussion. Ensuite, en séance plénière, ils ont écouté les experts présenter le fédéralisme belge (son histoire et sa dynamique politique) et leur ont posé des questions. Après cela, les citoyens se sont réunis à nouveau en groupes de discussion, avant de rencontrer les deux politiciens qui ont livré une courte intervention (20 minutes chacun) suivie d'une séance de questions et réponses. Une troisième discussion en petits groupes a clos la rencontre. Les échanges ont été enregistrés et retranscrits in extenso. Il en résulte deux 
corpus de discours citoyens, l'un en français (corpus-FR, 52003 mots) et l'autre en néerlandais (corpus-NL, 47579 mots).

Une question importante est évidemment de savoir dans quelle mesure les interventions des politiciens et des experts ont pu influer sur les participants. Une recherche précédente (Reuchamps, Charlier, 2011) a démontré que leurs préférences (mesurées par un pré- et post-questionnaire) ont, pour certains d'entre eux, évolué au cours de la rencontre. Mais la nature de ces changements est différente. Certains participants étaient peu au fait de la politique belge et leur participation à ces discussions leur a permis de se forger leur propre opinion. D'autres avaient déjà une opinion, mais qui, au fil des échanges, s'est révélée en décalage avec la dynamique actuelle du fédéralisme, ce qui les a amenés à la modifier. Dans ces évolutions, l'influence des politiciens et des experts n'est pas directe, mais leurs interventions ont permis aux participants de consolider leur propre opinion.

Au-delà de la question de l'impact politique se pose la question de l'impact linguistique, c'est-à-dire dans quelle mesure les mots (et plus spécifiquement les métaphores) utilisés par les participants sont inspirés de ceux que les experts et les politiciens ont pu utiliser. Tout d'abord, il convient de signaler qu'aucune instruction linguistique ne leur avait été donnée en ce qui concerne l'usage ou non de métaphores. La comparaison des interventions des experts et des politiciens, d'une part, et des participants, d'autre part, ne révèle pas une forte circulation des métaphores. Si certaines expressions comme «coquille vide » ont été reprises par les participants pour parler de l’État fédéral, aucune trace significative d'impact linguistique n'a été détectée. Comme nous le verrons ci-dessous, il y a eu une plus forte circulation de métaphores entre les participants d'un même groupe qu'entre les experts et les politiciens, d'une part, et les participants, d'autre part. La raison se trouve probablement dans la durée et l’intensité des échanges entre les participants.

\section{Identification des métaphores}

Afin d'évaluer dans quelle mesure les citoyens utilisent des métaphores pour parler du fédéralisme belge, nous avons appliqué une version révisée de la Metaphor Identification Procedure (MIP) développée par le Groupe Pragglejazz (2007) selon les quatre étapes suivantes. Nous avons tout d'abord lu les deux corpus dans leur entièreté afin de nous faire une idée globale de leurs contenus respectifs. De ces deux corpus, nous avons, dans un deuxième temps, extrait automatiquement les données pertinentes en effectuant une recherche de concordances (1) pour les lemmes se référant directement au domaine cible du fédéralisme belge (voir le tableau 1 pour un aperçu des mots inclus dans cette recherche de concordance) et (2) pour les signaux lexicaux explicitant des croisements entre deux domaines, par exemple les mots qui pourraient 
Tableau 1. Lemmes faisant référence au domaine cible du fédéralisme belge utilisés pour l'extraction automatique d'occurrences pertinentes dans les deux corpus citoyens

\begin{tabular}{l|l}
\hline Corpus & Lemmes faisant référence au domaine cible du fédéralisme belge \\
\hline Corpus-FR & $\begin{array}{l}\text { Belg^, fédér }{ }^{\star}, \text { nation, pays, État, entité fédérée } \\
\text { Nom + belge, + communautaire, + fédéral, + fédéré, + institutionnel, + national }\end{array}$ \\
\hline Corpus-NL & $\begin{array}{l}\text { Belg*, feder }{ }^{\star}, \text { land, natie, land, staat, deelstaat } \\
\text { Nom + Belgisch, + communautair, + federaal, + institutioneel, + nationaal }\end{array}$ \\
\hline
\end{tabular}

explicitement introduire des expressions utilisées métaphoriquement (comme, (zo) als, comparer, vergelijken, symboliser, symboliseren...). Cette recherche de concordance a permis d'identifier respectivement 492 occurrences pertinentes dans le corpus-FR et de 495 dans le corpus-NL.

Les contextes pertinents ont ensuite été analysés qualitativement pour identifier les expressions utilisées métaphoriquement. Les contextes analysés comptaient 150 caractères à gauche et à droite du mot ciblé. À cette étape, il convient de souligner que, comme nous nous sommes intéressés à la façon dont les citoyens parlent du fédéralisme belge, nous n'avons pas évalué le potentiel métaphorique de chaque unité lexicale, mais seulement des unités lexicales utilisées pour se référer au fédéralisme belge, ce qui nous distingue de la MIP classique. Cela implique, par exemple, que nous n'avons pas pris en compte les diverses utilisations métaphoriques du mot «Belgique», s'il n'était pas utilisé en lien avec le fédéralisme belge. Par contre, pour l'identification métaphorique proprement dite, nous avons suivi la méthodologie MIP, en comparant la signification du mot dans le contexte avec ses significations de base (Pragglejaz Group, 2007). Comme suggéré par Steen et al. (2010), nous n'avons pas pris en compte la dimension historique pour établir les significations de base des mots analysés. Pour cette étape de l'analyse, nous avons respectivement utilisé Le Petit Robert de la langue française 2013 (édition en ligne) comme dictionnaire de référence pour le corpus-FR et le Van Dale. Groot Woordenboek der Nederlandse Taal pour le corpus-NL. Cela a mené à l'identification de 99 contextes métaphoriques dans le corpus-FR (20,1\%) et 73 dans le corpus-NL $(14,8 \%)^{2}$.

2. Cette différence est statistiquement significative $\left(\chi^{2}=4.953, \mathrm{df}=1, \mathrm{p}<0.05\right)$. Cependant, comme l'objet de cet article n'est pas d'étudier les possibles différences culturelles et linguistiques dans l'usage des métaphores, aucune conclusion n'est tirée de cette observation. Il n'est reste pas moins que cette question mériterait d’être étudiée dans d'autres recherches. 
Tableau 2. Contextes métaphoriques et non métaphoriques dans le corpus-FR et le corpus-NL

\begin{tabular}{lcccc}
\hline & \multicolumn{2}{c}{ Corpus-FR } & \multicolumn{2}{c}{ Corpus-NL } \\
\hline & N & $\%$ & N & $\%$ \\
\hline Contextes métaphoriques & 99 & $20,1 \%$ & 73 & $14,8 \%$ \\
\hline Contextes non métaphoriques & 393 & $79,9 \%$ & 422 & $85,2 \%$ \\
\hline Total & 492 & $100 \%$ & 495 & $100 \%$ \\
\hline
\end{tabular}

La difficulté de se prononcer sur le caractère métaphorique de certaines unités lexicales peut être illustrée par les exemples (1) et (2) (voir également Perrez, Reuchamps, 2014). Dans l'exemple (1), un citoyen fait référence à la construction du fédéralisme belge en utilisant le terme "usine à gaz» pour dénoter sa structure complexe et son manque d'efficacité. Dans l'exemple (2), selon un autre citoyen, cette inefficacité peut être expliquée par la «wafelijzerpolitiek», c'est-à-dire la «politique du gaufrier » qui renvoie au principe de dépenser symétriquement pour une communauté ce qui est dépensé pour l'autre.

1. FR. [...] On va continuer à monter une usine à gaz (PBF, B 8, 249-25)

2. NL. Ik denk dat als men het woord wafelijzerpolitiek ook zei als wij al een projectportefeuille op ons werk hebben waar een Europese subsidie moet verdeeld worden. (PBN, K 6, 910-916)

FR. Je pense qu'on utilisait aussi le mot politique du gaufrier lorsque nous avions déjà un projet au boulot pour lequel une subvention européenne devait être partagée.

Ces deux expressions renvoient à des images très parlantes, respectivement une usine inefficace et un gaufrier, qui peuvent être apparentées à la métaphore conceptuelle générique L'ÉTAT EST UNE MACHINE. Cependant, ces deux unités sont clairement lexicalisées dans la mesure où elles apparaissent comme unités monosémiques dans les dictionnaires de référence et dans la mesure où leur unique sens attesté désigne respectivement les situations décrites par ces citoyens. Elles doivent par conséquent être traitées comme des expressions non métaphoriques dans ce contexte.

\section{Comptage des métaphores}

Une dernière remarque méthodologique concerne la manière de compter les unités métaphoriques. Puisque nous sommes principalement intéressés par la question de savoir comment les citoyens parlent du fédéralisme belge et non par le pourcentage total d'unités métaphoriques que recouvre notre corpus, nous avons adopté un système de comptage différent de celui préconisé par la 
procédure MIP, en mettant l'accent sur les contextes métaphoriques plutôt que sur les unités lexicales. L'exemple suivant illustre cette méthode de comptage.

3. FR. C'est comme dans un ménage, on ne règle jamais les solutions une fois pour toutes. On se marie, ou on vit ensemble, peu importe, à 20 ans, puis on a des enfants, puis les enfants deviennent grands, puis le bonhomme fait sa crise de la quarantaine, puis on se dit que tout compte fait, on se dit que c'était quand même pas si mal et puis rien, et puis entre-temps, madame est ménopausée et puis... Puis elle a perdu son job, puis les enfants se sont mariés, voilà que la maison est trop grande... les situations évoluent et je ne pense pas qu'on va rêver d'avoir une situation immuable. J'arrête les figures et les fables. (PBF, B 8, 1968-1977)

Ce passage est particulièrement intéressant, car ce participant a produit délibérément (comme il l'admet lui-même) de nombreuses métaphores du domaine de la famille pour parler du fédéralisme belge. Dans une perspective MIP, à partir de la deuxième phrase, chaque mot peut être considéré comme une unité métaphorique (sauf peut-être pour les deux dernières phrases). Cependant, lorsqu'on se concentre sur ce que ce citoyen évoque à propos du fédéralisme belge, il suggère simplement que les problèmes ne vont pas être résolus une fois pour toutes, mais que la Belgique, comme n'importe quel autre couple, passera par des moments de turbulence. Ce que nous retiendrons pour notre analyse, c'est que le participant compare la Belgique à un couple. C'est pour cette raison que nous avons compté l'ensemble du passage comme une métaphore, même s'il serait sans doute plus exact de parler de contextes métaphoriques que de métaphore. Les exemples similaires ont été traités de la même manière. Nous pensons que cette façon de compter les contextes métaphoriques nous permet d'éviter les biais en faveur de certains domaines conceptuels (dans ce cas, le domaine conceptuel de la famille).

Les analyses de ces deux corpus ont été effectuées indépendamment par les deux auteurs. Les cas problématiques ont été discutés par la suite pour parvenir à un accord. Les résultats de cette analyse quantitative sont présentés dans la partie qui suit.

\section{Résultats}

\section{Analyses préliminaires}

Le tableau ci-dessous résume les résultats globaux de notre étude. Il est difficile d'envisager des comparaisons quantitatives précises avec des études antérieures (comme celle de Krennmayr, 2011) dans la mesure où nous n'avons pas pris en compte la nature métaphorique de chaque unité lexicale du corpus. Cependant, la proportion de métaphores dans les discours citoyens est assez élevée, ce qui semble suggérer que les citoyens, lorsqu’ils sont invités 
Tableau 3. Distribution des métaphores à travers les domaines conceptuels dans les corpus des citoyens francophones (corpus-FR) et néerlandophones (corpus-NL)

\begin{tabular}{l|c|c|c}
\hline Domaine source & Nombre de contextes métaphoriques & Total \\
\hline construction & Corpus-FR & Corpus-NL & \\
\hline personnification & 15 & 33 & 48 \\
\hline machine & 16 & 11 & 27 \\
\hline voyage & 16 & 5 & 22 \\
\hline famille & 12 & 6 & 22 \\
\hline maladie & 5 & 4 & 16 \\
\hline entreprise & 1 & 2 & 7 \\
\hline entité & 1 & 6 & 7 \\
\hline instrument & 4 & 4 & 5 \\
\hline conteneur & 4 & 1 & 5 \\
\hline nourriture & 4 & 1 & 5 \\
\hline théâtre & 2 & 0 & 4 \\
\hline laboratoire & 1 & 0 & 2 \\
\hline vêtements & 1 & 0 & 1 \\
\hline Total & 99 & 0 & 1 \\
\hline & & 73 & 172 \\
\hline
\end{tabular}

à parler du fédéralisme belge, ont tendance à recourir aux métaphores pour exprimer leur perception et compréhension de ce concept politique abstrait.

Dans les sections suivantes, nous nous concentrerons sur l'utilisation des métaphores par les citoyens, en considérant plus particulièrement les domaines sources sur lesquels elles sont construites. À cette fin, nous avons suivi les recommandations méthodologiques de Cameron (2007, p. 205) :

Researchers adopting a discourse approach to metaphor have to accept that it is not possible to come up with a limited set of categories into which each linguistic metaphor can be reliably placed. [...] A principled flexibility to the grouping of linguistic metaphors appears to be the most suitable approach with discourse data.

En conséquence, nous nous sommes appuyés sur une approche bottom-up pour identifier les domaines pertinents en plaçant les métaphores dans des catégories conceptuelles plus larges. Comme nous nous intéressons à la façon dont les métaphores sont utilisées par les citoyens pour décrire le fédéralisme belge, nous présenterons les résultats de notre analyse quantitative globalement, sans faire aucune autre distinction entre groupes de citoyens. Nous discuterons cependant des différences interlinguistiques quand celles-ci nous sembleront pertinentes. 


\section{Domaines conceptuels}

Les domaines conceptuels les plus pertinents provenant des discours citoyens sont résumés dans le tableau 3 ci-dessus. Ces résultats montrent que lorsqu'ils parlent métaphoriquement du fédéralisme belge, les citoyens utilisent fréquemment des métaphores liées au domaine de la construction, de la personnification, de la machine, du voyage, de la famille et, dans une moindre mesure, de la maladie et de l'entreprise. Les domaines de la construction, de la machine et du voyage sont les domaines sources les plus fréquents pour évoquer le fédéralisme belge, avec les cas de personnifications.

Le domaine de la construction constitue le domaine prédominant dans la perception citoyenne du fédéralisme belge. Comme cela a été souvent souligné (voir, entre autres, Schäffner, 1996; Musolff, 2004; Elvert, 2006), ce domaine, qui dérive de la métaphore conceptuelle primaire UNE ORGANISATION EST UNE STRUCTURE PHYSIQUE (Grady, 1997), est particulièrement pertinent pour dénoter métaphoriquement les institutions politiques. Il est par exemple très conventionnel de parler de construction d'une structure politique ou de percevoir un État comme un immeuble divisé en de nombreux étages. Dans nos données, le nombre élevé de métaphores faisant référence à ce domaine s'explique notamment par l'occurrence fréquente d'extensions métaphoriques conventionnelles des unités lexicales structuur ("structure», voir les exemples 4 et 5), niveau (voir l'exemple 6) et, dans une moindre mesure, construire (voir l'exemple 7). Globalement, pour référer métaphoriquement au fédéralisme belge, ce domaine est plus fréquemment utilisé par les citoyens néerlandophones que par les francophones (voir tableau 3). Une explication possible de cette différence est l'emploi plus fréquent du mot structuur en néerlandais (28 fois) que du mot structure en français (seulement 6 fois). Ainsi, les citoyens apparaissent plus enclins à parler de structure fédérale en néerlandais et de système fédéral en français, alors qu'ils réfèrent au même objet.

4. FR. Un État fédéral, une structure fédérale, ça peut être très différent, ça peut être une structure fédérale forte ou une structure fédérale résiduaire. (PBF, D 8 , 2336-2337)

5. NL. Die solidariteit moet je buiten de Belgische structuur zien. (PBN, L 6, 1522 1523)

FR. Cette solidarité doit être considérée en dehors de la structure belge.

6. NL. [...] over het feit dat het Belgisch niveau overbodig is. (PBN, $L 1,1414$ )

FR. ... sur le fait que le niveau belge est superflu.

7. FR. [...] le fédéralisme tel qu'il a été construit progressivement (PBF, D 8, 38973898) 
Percevoir un pays comme une personne (L'ÉTAT EST UNE PERSONNE) a SOUvent été considéré comme l'une des métaphores politiques conceptuelles les plus conventionnelles (voir, par exemple, Lakoff, 1996, 2004). Il n'est donc guère surprenant de la retrouver dans les discours citoyens. Plus spécifiquement, comparer l'État à une personne permet de comprendre son fonctionnement plus concrètement (exemple 8).

8. FR. Et je pense que c'est ce qui est arrivé parce que quand la Flandre galérait en 1930, il était normal que la riche industrie sidérurgique wallonne alimente le pays. (PBF, B 8, 556-557)

Un autre domaine récurrent dans les données citoyennes est le domaine de la machine. La forte proportion de métaphores basées sur ce domaine conceptuel s'explique par l'utilisation fréquente des extensions métaphoriques du verbe fonctionner en français, et werken en néerlandais, pour désigner une organisation qui remplit sa fonction; cet usage représente plus de $50 \%$ des cas (voir les exemples 9 et 10). Dans l'ensemble, lorsque les citoyens comparent le fédéralisme belge à une machine (LE FÉDÉRALISME BELGE EST UNE MACHINE), ils expriment que le système politique est trop complexe et ne fonctionne pas correctement (voir les exemples 10 et 11). Notons que cette image est plus présente encore dans le corpus francophone que dans le corpus néerlandophone. Cette différence renvoie de manière inverse à celle du domaine de la construction. On trouve ici la même raison : pour désigner le même objet - le fédéralisme belge -, les francophones recourent plus souvent au domaine de la machine alors que pour les néerlandophones, il s’agit du domaine de la construction.

9. FR. Il faut distinguer le fédéralisme belge tel que nous le connaissons à l'heure actuelle, du fédéralisme tel qu'il a fonctionné dans les temps passés. (PBF, D 5 , 2469-2471)

10. NL. Maar ik denk dat je kunt concluderen dat het federalisme zoals het nu is dat het niet werkt. (PBN, N 4, 3318-3319)

Fr. Mais, je pense que l'on peut conclure que le fédéralisme, en l'état actuel, ne fonctionne pas.

11. FR. On a coupé le citoyen du fonctionnement d'une espèce de mécanisme, de machine folle lancée sur elle-même. (PBF, B 8, 839-840)

Les métaphores du voyage constituent également un domaine conceptuel fréquent, mais assez diversifié, présentant le fédéralisme belge tantôt comme une entité sur un chemin (exemple 12), tantôt comme une escale sur un chemin (exemple 13) ou encore comme une destination à atteindre (exemples 14 et 15). Ces métaphores renvoient à la métaphore conceptuelle LA POLITIQUE EST UN VOYAGE, qui a souvent été identifiée comme une métaphore centrale dans notre façon de parler de politique (voir, par exemple, Arcimaviciene, 2007; Charteris-Black, 2011 ; Cibulskienè, 2012). Dans l'ensemble, ces métaphores 
sont plus fréquentes dans le corpus francophone que dans le corpus néerlandophone. Cette différence est politiquement intéressante, car elle peut s'expliquer par le sentiment partagé par plusieurs citoyens francophones que le fédéralisme ne serait qu'une étape dans l'évolution de la Belgique vers sa scission, ce qu'ils redoutent (Reuchamps, 2009, 2013).

12. FR. Je crois qu'à partir du moment où le fédéralisme est évolutif, il ira de crises en crises. (PBF, D 8, 2561)

13. FR. Il dit que la Belgique, c'est une étape qui est devenue non nécessaire. (PBF, $\mathrm{M}, 780-781)$

14. NL. doordat men maar in zeer kleine stapjes tot dat Belgisch federalisme is gekomen (PBN, K 1, 230-231)

FR. Parce qu'on n'est arrivé qu'à très petits pas au fédéralisme belge

15. NL. Die discussie over de toekomst van het Belgisch federalisme, waar we naartoe moeten. (PBN, M, 1219-1220)

FR. Cette discussion sur le futur du fédéralisme belge est de savoir où nous devons aller.

Le cinquième domaine est le domaine de la famille. Bien qu'il ne soit pas prédominant en termes d'occurrences, il apparaît qualitativement comme un domaine conceptuel important. Felstiner (1983) avait déjà mis en avant l'usage des métaphores de la famille dans les discours patriotiques, mais le rôle des relations familiales dans la façon dont les citoyens s'approprient des processus politiques complexes a été illustré par Lakoff (1996) en décrivant comment différents modèles parentaux («strictfather » vs «nurturantparent») pouvaient façonner notre perception de certains phénomènes politiques. L'influence conceptuelle des relations familiales a été confirmée par plusieurs chercheurs dont Hayden (2003), qui a étudié la notion de «maternal politics» (en opposition à une "strict father morality»), et par McAdams et al. (2008), qui ont mis en évidence l'importance des métaphores de la famille dans les récits de vie.

La pertinence de ce domaine conceptuel ressort également fortement de l'analyse de notre corpus. En effet, tandis que dans les autres domaines conceptuels, les métaphores ne révèlent pas de vues divergentes sur le fédéralisme belge, les métaphores liées à la famille montrent, en revanche, un haut niveau d'opposition (voir l'exemple 3 ci-dessus et les exemples 16 à 18 ci-dessous). Les citoyens décrivent le fédéralisme belge à travers le prisme de la métaphorele fÉdÉRALISME BELGE EST UNE RELATION AMOUREUSE. Cette métaphore trouve ses racines dans la métaphore basique L'ÉTAT EST UNE PERSONNE (voir ci-dessus). Dans un pays fédéral reposant sur deux communautés principales, il semble presque naturel de percevoir ce même État comme deux personnes interagissant ensemble. La métaphore LE FÉdÉRALISME BELGE EST UNE RELATION AMOUREUSE ne renvoie pas directement aux métaphores de la famille en termes de modèles parentaux tels que discutés précédemment. Cependant, 
comme les relations entre les communautés belges sont présentées en termes de couple, de mariage et de divorce dans nos données et puisque ces relations amoureuses entre deux personnes sont centrales pour la dynamique familiale, il nous semble que cette métaphore puisse être considérée comme une extension naturelle du domaine conceptuel de la famille.

Qui plus est, les opinions politiques derrière la métaphore LE FÉDÉRALISME BELGE EST UNE RELATION AMOUREUSE varient largement. Le fédéralisme belge est ainsi vu comme une relation amoureuse par les citoyens qui plaident pour l'unité de la Belgique. D'autres citoyens voient le fédéralisme belge comme un mariage de raison et souhaitent un fédéralisme qui reconnaisse les différences entre les néerlandophones et les francophones, mais vise aussi à construire un avenir commun. Un troisième groupe croit en une relation « living apart together» (LAT ou, en français, vivre ensemble chacun chez soi), ce qui signifie que les deux principaux groupes linguistiques devraient rester dans le même pays, mais vivre leur propre vie (voir l'exemple 18). Un dernier groupe, au contraire, voit la Belgique comme un mariage forcé et invite à sa rupture (voir la discussion présentée dans le tableau 4). Du point de vue de la science politique, c'est un constat intéressant : les métaphores sont donc bien plus que de simples figures rhétoriques, car elles renvoient à des visions politiques différentes.

16. FR. Si l'on compare avec un ménage, certains ménages se marient avec contrat de mariage, d'autres pas. (PBF, B 1, 188-190)

17. FR. [...] parce que je ne sais pas comment cohabitent la région wallonne et la région flamande avec le fédéralisme, etc. (PBF, B 6, 145-148)

18. NL. Normale partijen die een staatshervorming willen enzovoort die willen eigenlijke hetzelfde als we zo zeggen een ernstige $L A T$ relatie in dit land. (PBN, M 5 , 3130-3131)

FR. Les partis normaux qui veulent une réforme de l'État, veulent en fait la même chose que, disons, une « relation LAT» sérieuse dans ce pays.

Cette notion de relation amoureuse semble jouerun rôle particulièrement important dans la façon dont les citoyens construisent leur compréhension des relations entre les deux principales communautés du pays. Plus qu'une figure de style, cette métaphore de la relation amoureuse permet de refléter les différentes visions du fédéralisme belge et, ce faisant, offre un point de référence conceptuelle particulièrement pertinent permettant aux citoyens d'exprimer leurs propres représentations. Ceci est clairement illustré dans l'extrait suivant (repris dans le tableau 4) dans lequel trois citoyens expriment leur point de vue divergent sur le fédéralisme belge avec un enchaînement de métaphores relatives au concept de mariage. 
Tableau 4. Discussion structurée autour de la métaphore du mariage (corpus-NL)

\begin{tabular}{|c|c|}
\hline Citoyens néerlandophones & Traduction française \\
\hline $\begin{array}{l}\mathrm{L} 2 \text { : Het is vergelijken met dat huwelijk he. } \\
\text { De Belgische staat is een gearrangeerd en } \\
\text { geforceerd huwelijk geweest (2263-2266) } \\
{[\ldots]}\end{array}$ & $\begin{array}{l}\text { L } 2: \text { :ll est comparé à ce mariage, non? } \\
\text { L'État belge a été un mariage arrangé et } \\
\text { forcé [...] }\end{array}$ \\
\hline $\begin{array}{l}\mathrm{L} 6: \text { Het is inderdaad een gearrangeerd } \\
\text { huwelijk en het is gearrangeerd door de } \\
\text { internationale gemeenschap (2268-2269) } \\
{[\ldots]}\end{array}$ & $\begin{array}{l}\text { L } 6 \text { : C'est en effet un mariage arrangé } \\
\text { et il a été arrangé par la communauté } \\
\text { internationale [...] }\end{array}$ \\
\hline $\begin{array}{l}\text { L } 6 \text { : Een gearrangeerd huwelijk kan } \\
\text { ook ontbonden worden, zo moeilijk is } \\
\text { dat allemaal niet. Het moet gewoon } \\
\text { erkend worden door de internationale } \\
\text { gemeenschap (2279-2280) }\end{array}$ & $\begin{array}{l}\text { L } 6: \text { Un mariage arrangé peut aussi être } \\
\text { abrogé, ce n'est pas si difficile. Cela doit } \\
\text { juste être accepté par la communauté } \\
\text { internationale }\end{array}$ \\
\hline $\begin{array}{l}\text { L } 2 \text { : Ja maar dat is getrouwd voor goede en } \\
\text { kwade dagen en wij zijn nu in kwade dagen. } \\
(2281-2282)\end{array}$ & $\begin{array}{l}\text { L } 2 \text { : Oui, mais il a été marié pour le meilleur } \\
\text { et pour le pire et nous sommes maintenant } \\
\text { dans la période la plus difficile. }\end{array}$ \\
\hline $\begin{array}{l}\text { L } 6 \text { : Maar bij een gearrangeerd huwelijk is } \\
\text { het niet in goede en kwade dagen vrijwillig, } \\
\text { maar is het verplicht in kwade dagen. [...] ik } \\
\text { hoop toch dat we zover zijn dat huwelijken } \\
\text { niet meer verplicht zijn ofwel? (2283-2287) }\end{array}$ & $\begin{array}{l}\text { L } 6 \text { : Mais dans un mariage arrangé, ce } \\
\text { n'est pas volontairement pour le meilleur } \\
\text { ou pour le pire, mais obligatoirement pour } \\
\text { le pire. J'espère quand même que nous } \\
\text { sommes arrivés à une situation où les } \\
\text { mariages ne sont plus forcés, n'est-ce pas? }\end{array}$ \\
\hline $\begin{array}{l}\text { L } 1 \text { : Neen, maar je kan dan toch karakter } \\
\text { tonen, karakter tonen. (2288) }\end{array}$ & $\begin{array}{l}\text { L } 1 \text { : Non, mais vous pouvez toujours faire } \\
\text { preuve de caractère. }\end{array}$ \\
\hline $\begin{array}{l}\text { L } 6 \text { : Als ons dat ieder jaar } 10 \text { miljard euro } \\
\text { kost, vind ik dat toch... (2289) }\end{array}$ & $\begin{array}{l}\text { L } 6 \text { : Si cela nous coûte } 10 \text { milliards d'euros } \\
\text { par an, je trouve que... }\end{array}$ \\
\hline
\end{tabular}

En plus d'illustrer le rôle de la métaphore du mariage sur les représentations citoyennes, ce passage est un bel exemple de la façon dont l'emploi d'une métaphore par un citoyen peut cadrer les réactions d'autres citoyens. Cette fonction spécifique des métaphores dans la communication a été décrite par Steen (2008, p. 230, notre traduction) comme la création d'une «base commune de référence», qui selon lui se produit notamment «quand des sujets problématiques doivent être évoqués par les interlocuteurs».

Un autre domaine conceptuel relativement fréquent est celui de la maladie (LE fÉdÉralisme beLGe eSt Une malAdIE). Ainsi, dans les exemples 19 à 22, le fédéralisme belge est présenté respectivement comme une maladie (exemples 19 et 20), une excroissance infectieuse (exemple 21) ou une maladie de riches (exemple 22).

19. NL. [...] en dat dat is de ziekte van het federalisme. Ik heb dat niet voor niks daarstraks een noodzakelijk kwaad genoemd. (PBN, M 1, 3069-3070) 
FR. [...] et ça, c'est la maladie du fédéralisme. Je ne l'ai pas appelé pour rien un mal nécessaire.

20. NL. Welke bevoegdheden op het nationale niveau, welke bevoegdheden op het regionale niveau? Het wordt helemaal een pest bij wijze van spreken. (PBN, M, 2533-2534)

FR. Quelles compétences au niveau national ? Quelles compétences au niveau régional ? C'est devenu un fléau pour ainsi dire.

21. FR. On a été créer un espèce de furoncle qui s'appelait Belgique... (PBF, B 8, 1097-1098)

22. FR. Le fédéralisme est au départ le résultat d'un égocentrisme et d'une maladie de riches. (PBF, $\mathrm{B} 8,134-136)$

Un dernier domaine conceptuel utilisé pour parler du fédéralisme belge est le domaine des entreprises (LE FÉdÉRALISME BELGE EST UNE ENTREPRISE). LeS métaphores basées sur ce domaine source se révèlent être plus fréquemment utilisées par les citoyens néerlandophones que par les citoyens francophones. Avec cette métaphore, les citoyens ont tendance à exprimer la façon dont ils comprennent le fonctionnement de l’État en général et plus spécifiquement la façon dont les politiciens dirigent le pays. Ces exemples illustrent également l'importance croissante du paradigme économique dans notre compréhension du processus politique, comme suggéré par Koller (2009).

23. NL. Hoe werkt een staat? Een beetje zoals een bedrijfsleider over zijn bedrijf. (PBN, K 4, 1232-1233)

FR. Comment fonctionne un État ? Un peu comme un chef d'entreprise avec son entreprise.

24. NL. Als je vergelijkt met een bedrijf, een bedrijf laat je ook niet leiden door de werkman of de kuisvrouw bij wijze van spreken. (PBN, L 3, 2327-2329)

FR. Si vous comparez avec une entreprise, vous ne laissez pas diriger une entreprise par un ouvrier ou une femme de ménage, pour ainsi dire.

\section{Discussion}

Notre étude, basée sur l'analyse de discussions de groupes sur le fédéralisme belge, visait à analyser les discours citoyens afin d'évaluer dans quelle mesure les citoyens utilisent des métaphores pour décrire les relations et les processus politiques complexes. Pour analyser les expressions métaphoriques dans les discours citoyens, nous avons développé une méthode reposant sur une version légèrement modifiée de la procédure MIP. De cette recherche, les enseignements suivants peuvent être tirés.

Tout d'abord, notre étude montre que les citoyens produisent de nombreuses métaphores lorsqu'ils sont invités à parler du fédéralisme belge. Cela suggère que l'usage des métaphores n'est pas limité aux discours des élites, 
mais que les citoyens expriment aussi les processus politiques de façon métaphorique. Cette première observation confirme l'hypothèse de Bougher (2012), à savoir que l'on peut tirer des enseignements précieux de l'étude des discours citoyens. À cet égard, les groupes de discussions semblent être une voie prometteuse pour recueillir des données pertinentes pour les discours citoyens, en particulier pour l'étude des métaphores.

Nos résultats suggèrent également que les citoyens utilisent différents domaines conceptuels pour évoquer le fédéralisme belge. Parmi cette variété de domaines conceptuels, on peut observer que les domaines conceptuels qui sont traditionnellement liés au discours politique, comme les domaines de la construction, de la famille, de la machine, du voyage ou la personnification, émergent également des discours citoyens, confirmant leur importance non seulement dans la façon dont nous parlons de politique, mais aussi dans la façon dont nous pensons la politique.

En nous concentrant sur le point de vue des citoyens sur le fédéralisme belge, on a pu remarquer que ces domaines sont fréquemment mobilisés pour refléter des images négatives du fédéralisme qui sont véhiculées par différentes métaphores comme celle d'une structure complexe, d'une machine qui ne fonctionne pas correctement, d'un mariage forcé ou d'une maladie. Cependant, ces différents domaines ne sont pas simplement des façons différentes de dire la même chose. Le choix d'un domaine source particulier comporte des conséquences conceptuelles. Ainsi, parler de la Belgique comme d'une structure complexe ou d'une machine déficiente permet de souligner la façon dont les différentes couches du fédéralisme belge ont été mises en place et comment ces différents niveaux politiques interagissent (Caluwaerts, Reuchamps, 2015). C'est une autre conception du fédéralisme belge qui est transmise lorsqu'on compare la Belgique à une relation amoureuse qui souligne plutôt les liens qu'entretiennent les deux principaux groupes linguistiques (voir, pour aller plus loin, une étude de l'impact de la métaphore du Tetris sur la représentation du fédéralisme belge, Perrez, Reuchamps, 2015). Pour l'étude des discours sur le fédéralisme belge, sujet qui compte politiquement dans ce pays, c'est une découverte importante, car l'emphase sur la structure ou le fonctionnement appelle à des changements en termes de répartition de pouvoirs (entre les différents niveaux de gouvernement) alors que l'emphase sur la relation amoureuse appelle plutôt à des changements en termes de relations inter-communautaires (c'est-à-dire dans les relations entre néerlandophones et francophones).

En outre, on peut avancer que le domaine familial semble être particulièrement pertinent pour la compréhension et le cadrage des discours citoyens dans le contexte politique belge. Cet enseignement découle de l'observation que la plupart des métaphores familiales ont tendance à cadrer des passages entiers de discussions (voir l'exemple 3 ou le tableau 4). Dans ce contexte, basé sur deux communautés linguistiques souvent présentées comme constamment 
opposées l'une à l'autre, ces relations familiales se matérialisent par des métaphores liées à une relation amoureuse qui peut prendre différentes formes : une relation "LAT», ou encore un couple cohabitant ou un mariage d'amour. Il est intéressant d'observer que la diversité des formes de relations amoureuses envisageables permet aux citoyens d'exprimer une variété de perceptions à l'égard du fédéralisme belge. Nos données montrent qu'ils n’hésitent pas à utiliser de telles métaphores pour faire part de leurs perceptions et préférences politiques.

Un autre élément intéressant qui ressort de l'analyse des domaines conceptuels mobilisés par les citoyens pour produire des métaphores est la similitude entre les deux groupes linguistiques. Les francophones comme les néerlandophones ont tendance à recourir aux mêmes domaines conceptuels pour parler du fédéralisme belge. Cela ne signifie pas nécessairement qu'ils ont la même vision sur ses fonctions et son avenir, mais que les domaines conceptuels qu’ils utilisent pour donner du sens à cette réalité sont partagés. La comparaison de la fréquence de ces domaines conceptuels révèle néanmoins quelques différences. Ainsi, les citoyens néerlandophones ont tendance à évoquer l'État belge en termes de structure fédérale alors que les citoyens francophones le font en termes de système fédéral. Cette différence semble renvoyer à des représentations potentiellement différentes de la nature de l'État. Cette question devrait dès lors être explorée plus en profondeur afin d'étudier si des différences existent dans la conception de l'État entre les deux communautés et, le cas échéant, de comprendre quels sont les facteurs qui pourraient les motiver.

En considérant nos données et plus spécifiquement les différentes métaphores qui ont été identifiées, on peut être frappé par la nature communicationnelle différente des métaphores produites par les citoyens. Alors que certaines métaphores semblent avoir été produites explicitement pour convaincre un interlocuteur ou expliquer un point de vue (voir l'exemple 3 ou le tableau 4), d'autres métaphores constituent des moyens conventionnels pour parler de politique. Cette distinction peut être illustrée par deux exemples déjà cités (reproduits ici pour en faciliter la lecture).

25. FR. [...] le fédéralisme tel qu'il a été construit progressivement... (PBF, D 8, 38973898)

26. FR. [...] parce que pour moi la Belgique reste une espèce de grande famille, malgré tout. (PBF, D 1, 2289-2290)

Dans ces deux exemples, la Belgique est présentée métaphoriquement en termes, d'une part, de structure physique (exemple 25) et, d'autre part, de famille (exemple 26). Alors que sur le plan conceptuel, ces deux métaphores sont des exemples de métaphores conceptuelles fréquemment utilisées dans les discours politiques (respectivement UNE ORGANISATION EST UNE STRUCTURE PHYSIQUE et L'ÉTAT EST UNE FAMILLE), elles jouent un rôle communication- 
nel différent. Avec la métaphore de la famille dans l'exemple 26, le citoyen D 1 présente explicitement sa propre conceptualisation de la Belgique. Ce n'est pas le cas dans l'exemple 25, où la métaphore de la construction est uniquement exprimée indirectement, par l'utilisation métaphorique conventionnelle du verbe construire.

La nécessité de faire cette distinction entre les métaphores nouvelles et les métaphores conventionnelles a été soulignée par Steen (2008), qui a proposé un modèle tridimensionnel d'analyse des métaphores dans le discours. Le cadre analytique repose sur la distinction entre différents niveaux, à savoir le niveau linguistique (métaphores directes vs indirectes), le niveau conceptuel (métaphores nouvelles vs conventionnelles) et le niveau communicatif (métaphores délibérées vs non délibérées). Si on applique le modèle de Steen (2008) à nos données, la métaphore de la famille, dans l'exemple 26, doit être considérée comme directe, nouvelle et délibérée, alors que la métaphore de la construction comme indirecte, conventionnelle et non délibérée. Bien que la pertinence de cette distinction entre métaphore délibérée et non délibérée fasse l'objet de débats dans la littérature (voir par exemple Gibbs, 2011), elle semble utile dans l'analyse du discours politique. En effet, cette distinction permet de mettre le doigt sur des divergences non négligeables dans la perception citoyenne de l'État. Dans nos exemples, on pourrait avancer que la métaphore de la famille semble plus ancrée dans la conscience politique du citoyen que la métaphore de la construction. En outre, cette distinction pourrait nous aider à comprendre (1) pourquoi les acteurs politiques utilisent des métaphores - et quelles métaphores - dans leurs discours et (2) comment ils perçoivent et conceptualisent des notions complexes telles que la structure de l'État et les interactions entre ses entités. Ces questions devront être analysées dans de futures recherches.

\section{Pistes de recherche}

Cet article sur l'utilisation des métaphores par les citoyens belges constitue une première étape vers une approche plus globale de l'utilisation des métaphores politiques par les citoyens et plus précisément de leur impact politique. Dans l'analyse des métaphores à partir de discours politiques et médiatiques, cet impact politique est généralement pris pour argent comptant. Cependant, une compréhension plus fine de la nature de cet impact politique fait souvent défaut dans les recherches actuelles. Koller (2009, p.121) indique ainsi :

Metaphor helps construct particular aspects of reality and reproduce (or subvert) dominant schemas. The models of reality held and reinforced by groups with most power and discourse access then become quantitatively and qualitatively salient. In a cyclical fashion, discourse recipients are therefore more likely to encounter such 
hegemonic mental models underlying discourse, and given similar conditions of reception, such repeated exposure is likely to anchor and reinforce such models even further.

Pour être en mesure d'appréhender la façon dont les métaphores, à travers les discours, façonnent - activement ou non - la réalité politique, il est important d'étudier les relations entre les discours et leur environnement. En conséquence, deux axes de recherche spécifiques semblent être particulièrement prometteurs pour le développement des recherches sur la métaphore dans les discours politiques.

Le premier axe porte sur l'étude systématique de la percolation des métaphores à travers différents genres de discours politique (par exemple, des discours des élites aux discours des citoyens à travers les discours des médias) afin d'évaluer dans quelle mesure une métaphore circule d'un genre à l'autre et quelles directions ces métaphores suivent. Cette question de recherche soulève plusieurs autres questions, telles que : comment les métaphores politiques atteignent-elles les citoyens? Est-ce que les métaphores politiques circulent toujours du haut vers le bas (des discours des élites ou des médias vers les discours citoyens) ou y a-t-il d'autres moyens de diffusion des métaphores? Quel rôle jouent les médias dans ce processus de diffusion? Est-ce que les métaphores circulent au sein de groupes politiques différents (par exemple entre les citoyens)?

Une deuxième piste de recherche consiste à analyser l'impact des métaphores sur les citoyens. Bougher (2012, p.157, notre traduction) énonce en effet que les métaphores offrent « un mécanisme cognitif permettant d'expliquer comment les citoyens comprennent leur environnement politique à partir d'expériences et de connaissances non politiques ». Les métaphores ne reflètent donc pas seulement la manière dont la réalité est perçue, mais elles fonctionnent aussi comme des mécanismes par lesquels les citoyens parviennent à mieux comprendre des choix politiques et ainsi façonner leurs comportements politiques. Explorer plus profondément comment les métaphores influencent les citoyens peut contribuer à une meilleure compréhension du rôle et des fonctions qu'elles jouent dans les discours politiques et plus globalement dans nos interactions politiques quotidiennes.

\section{Références}

ARCIMAVICIEne Liudmila, 2007, "British politics today. Moral grounds as reflected in conceptual metaphor», Kalbotyra, $\mathrm{n}^{\circ} 57$ (3), p. 11-20.

BOUGHER Lori D., 2012, «The case for metaphor in political reasoning and cognition », Political Psychology, ${ }^{\circ} 33$ (1), p. 145-163. 
Calumaerts Didier, Reuchamps Min, 2015, "Combining federalism with consociationalism. Is Belgian consociational federalism digging its own grave? ", Ethnopolitics, $n^{\circ} 14$ (3), p. 277-295.

CAMERON Lynne, 2007, «Patterns of metaphor use in reconciliation talk», Discourse \& Society, $\mathrm{n}^{\circ} 18$ (2), p.197-222.

CAMERON Lynne, MASLEN Robert, 2010, «Using metaphor analysis to compare expert and public perceptions of the risk of terrorism», Metaphor Analysis. Research Practice in Applied Linguistics, Social Sciences and the Humanities, L. Cameron, R. Maslen éd., Londres, Equinox, p. 248-256.

Charteris-Black Jonathan, 2011, Politicians and Rhetoric. The Persuasive Power of Metaphor, Londres, Palgrave Macmillan.

CIBULSKIENÉ Jurga, 2012, "The development of the journey metaphor in political discourse. Time-specific changes », Metaphor and the Social World, $\mathrm{n}^{\circ} 2$ (2), p. 131-153.

De Cillia Rudolf, Reisigl Martin, Wodak Ruth, 1999, "The discursive construction of national identities », Discourse \& Society, nº 10 (2), p. 149-173.

De LANDTSheer Christ'l, 2009, "Collecting political meaning from the count of metaphor», Metaphor and Discourse, A. Musolff, J. Zinken éd., Houndmills, Palgrave Macmillan, p.59-78.

Deschoumer Kris, 2012, The Politics of Belgium. Governing a Divided Society, Houndmills, Palgrave Macmillan (Comparative Government and Politics Series).

Deschoumer Kris, Reuchamps Min, 2013, "The Belgian Federation at a crossroad», Regional \& Federal Studies, nº 23 (3), p. 261-270.

ELVERT Jürgen, 2006, « How many rooms are there in the European house?», L'Europe inachevée, M. Dumoulin, G. Duchenne éd., Bruxelles, Peter Lang, p. 127-142.

FELSTINER Mary Lowenthal, 1983, "Family metaphors. The language of an independence revolution », Comparative Studies in Society and History, n 25 (1), p. 154-180.

GIBBS Raymond W., 2011, "Are "deliberate" metaphors really deliberate? A question of human consciousness and action», Metaphor and the Social World, $n^{\circ} 1$ (1), p. 26-52.

GRAdY Joseph E., 1997, Foundations of Meaning. Primary Metaphors and Primary Scenes, PhD, Berkeley, University of California.

HAYDEN Sara, 2003, "Family metaphors and the Nation. Promoting a politics of care through the Million Mom March », Quarterly Journal of Speech, n 89 (3), p. 196-215.

KolLer Veronika, 2009, «Missions and empires. Religious and political metaphors in corporate discourse», Metaphor and Discourse, A. Musolff, J. Zinken éd., Houndmills, Palgrave Macmillan, p.116-134.

KREnNmAYR Tina, 2011, « Metaphor in newspapers», PhD, Amsterdam, Vrije Universiteit Amsterdam.

L'HôTE Émilie, 2010, "New Labour and globalization. Globalist discourse with a twist? », Discourse \& Society, n² 21 (4), p. 355-376.

- 2012, "Breaking up Britain"? Métaphores et discours sur la dévolution au Royaume-Uni», Les relations communautaires en Belgique. Approches politiques et linguistiques, J. Perrez, M. Reuchamps éd., Louvain-la-Neuve, AcademiaL’Harmattan (Science politique), p.161-189. 
LAKoff George, 1996, Moral Politics. How Liberals and Conservatives Think, Chicago, University of Chicago Press.

- 2004, Don't Think of an Elephant. Know your Values and Frame the Debate, White River Junction, Chelsea Green Publishing Company.

LAKOFF George, Johnson Mark, 1980, Metaphors We Live By, Chicago, University of Chicago Press.

McAdams Dan P., Albaugh Michelle, Farber Emily, Daniels Jennifer, Logan Regina L., OLSON Brad, 2008, «Family metaphors and moral intuitions. How conservatives and liberals narrate their lives », Journal of Personality and Social Psychology, $\mathrm{n}^{\circ} 95$ (4), p. 978-990.

Mercenier Heidi, Perrez Julien, Reuchamps Min, 2015, "Constructing Quebec and Wallonia. How political parties speak about their region», Minority Nations in Multinational Federations. A Comparative Study of Quebec and Wallonia, M. Reuchamps éd., Abingdon - New York, Routledge (Routledge Series in Federal Studies), p. 49-81.

Musolff Andreas, 2004, Metaphor and Political Discourse. Analogical Reasoning in Debates about Europe, Londres, Palgrave-Macmillan.

Perrez Julien, Reuchamps Min éd., 2012, Les relations communautaires en Belgique. Approches politiques et linguistiques, Louvain-la-Neuve, Academia-L'Harmattan (Science politique).

- 2014, "Deliberate metaphors in political discourse. The case of citizen discourse», Metaphorik.de, $\mathrm{n}^{\circ}$ 25, p. 7-41.

- 2015, "The "Belgian Tetris". Assessing the political impact of metaphors on citizens' perception of and attitude towards Belgian federalism », Metaphor and the social world, $\mathrm{n}^{\circ} 5$ (2), p. 223-244.

PRAGgLeJAZ Group, 2007, «MIP. A method for identifying metaphorically used words in discourse », Metaphor and Symbol, nº 22 (1), p.1-39.

REUCHAMPS Min, 2009, "Le fédéralisme et ses deux sens - négatifs - en Belgique francophone », Revue de la Faculté de droit de l'Université de Liège, n 54 (4), p. 527-540.

- 2011, L'avenir du fédéralisme en Belgique et au Canada. Quand les citoyens en parlent, Bruxelles, PIE-Peter Lang (Diversitas).

- 2013, "The future of Belgian Federalism through the eyes of the citizens », Regional \& Federal Studies, $\mathrm{n}^{\circ} 23$ (3), p. 353-368.

Reuchamps Min, Charlier Nathan, 2011, "Can you turn tomorrow Regionalist if you were yesterday Federalist? A qualitative assessment of federal preferences' changes in Francophone Belgium », 6th European Consortium for Political Research General Conference, Reykjavik.

Reuchamps Min, Perrez Julien, 2012, "Métaphores conceptuelles dans les discours citoyens en Belgique », Les relations communautaires en Belgique. Approches politiques et linguistiques, J. Perrez, M. Reuchamps éd., Louvain-la-Neuve, Academia-L'Harmattan (Science politique), p. 133-158.

SCHÄFFnER Christina, 1996, «Building a European house? Or at two speeds into a dead end? Metaphors in the debate on the United Europe », Conceiving of Europe. Diversity in Unity, A. Musolff, C. Schaeffner, M. Townson éd., Aldershot, Ashgate, p. 31-59. 
Semino Elena, 2008, Metaphor in Discourse, Cambridge, Cambridge University Press.

STEEN Gerard J., 2008, «The paradox of metaphor. Why we need a three-dimensional model of metaphor », Metaphor and Symbol, n 23 (4), p. 213-241.

Steen Gerard J., Dorst Aletta G., Herrmann J. Berenike, KaAl Anna, Krennmayr Tina, PASMA Trijntje, 2010, A Method for Linguistic Metaphor Identification. From MIP to MIPVU, Amsterdam, John Benjamins (Converging Evidence in Language and Communication Research). 